remove this type of myxoma once it is diagnosed. During surgical procedures, careful measures should be taken to prevent fragments of the tumor from breaking away.

\section{References}

1. Reynen K. Cardiac myxomas. New Engl J Med. 1996;333:1610-7.
2. MacGowan SW, Sidhu P, Aherne T, et al. Atrial myxoma: national incidence diagnosis and surgical management. Ir J Med Sci. 1993;162:223-6.

3. Roschkov S, Rebeyka D, Mah J, et al. The dangers of cardiac myxomas. Prog Cardiovasc Nurs. 2007;22:27-30.

4. Vogt PR, Jenni R, Turina MI. Infected left atrial myxoma with concomitant mitral valve endocarditis. Eur J Cardiothorac Surg. 1996;10:71-3.

5. Revankar SG, Clark RA. Infected cardiac myxoma: case report and literature review. Medicine. 1998;77:337-44.

\title{
Familial fetal-type rhabdomyoma of the tricuspid valve in the neonate: Malignant course for a benign disease
}

Francesca Viscardi, MD, ${ }^{\mathrm{a}}$ Gabriella Errico, MD, ${ }^{\mathrm{b}}$ Nicola Schiavo, MD, ${ }^{\mathrm{c}}$ Paolo Biban, MD, ${ }^{\mathrm{b}}$ Alessandro Mazzucco, MD, ${ }^{\mathrm{a}}$ and Giovanni Battista Luciani, MD, ${ }^{\text {a }}$ Verona, Italy

\section{Supplemental material is available online.}

We report 2 brothers with prenatal diagnosis of tricuspid valve masses, both requiring aggressive intensive care resuscitation shortly after birth and surgical excision of the tumor, which proved to be a fetal-type rhabdomyoma. The unique pathologic and clinical features of this rare disease are discussed.

\section{CLINICAL SUMMARY}

Patient 1. The first child had a critical neonatal period due to severe tricuspid valve stenosis, caused by 3 obstructive cardiac masses and severe pulmonary hypertension. Treatment included balloon atrial septostomy to maintain cardiac output and mechanical ventilation, with prostaglandin E1 (PGE1) infusion and inhaled nitric oxide (NO) for 2 months after birth. Discharge therapy with sildenafil and bosentan was continued until the time of elective surgical resection of tricuspid valve masses (Figure 1, A), at 11 months of age. Histology showed only dystrophic calcification. Excellent tricuspid valve function and no recurrence of cardiac

\footnotetext{
From the Divisions of Cardiac Surgery, ${ }^{\mathrm{a}}$ Pediatrics, ${ }^{\mathrm{b}}$ and Pathology, ${ }^{\mathrm{c}}$ University of Verona, Verona, Italy.

Received for publication Nov 17, 2007; accepted for publication March 23, 2008.

Address for reprints: Giovanni Battista Luciani, MD, Division of Cardiac Surgery,

University of Verona, O. C. M. Piazzale Stefani 1, Verona, 37126, Italy (E-mail:

gbluciani@yahoo.com).

J Thorac Cardiovasc Surg 2009;137:751-3

$0022-5223 / \$ 36.00$

Copyright (c) 2009 by The American Association for Thoracic Surgery

doi:10.1016/j.jtcvs.2008.03.066
}

masses were documented at follow-up echocardiogram 1 year after operation. The child has no clinical nor laboratory evidence of tuberous sclerosis.

Patient 2. The younger brother also had severe tricuspid valve obstruction (functional atresia), due to a huge tricuspid valve mass, and pulmonary hypertension at birth, with echocardiographic evidence of adequate right-to-left shunting at the atrial septal level. Mechanical ventilation, PGE1 infusion, and inhaled NO failed to stabilize clinical status, and emergency surgical resection was required at 21 days of life (Figure 1,B). Despite satisfactory repair of tricuspid valve, extracorporeal membrane oxygenation (ECMO) support was necessary for refractory hypoxia. Six days after ECMO weaning, the neonate died due to sepsis and recurrent respiratory failure. Histology of the lesion evidenced fibromyxoid stroma and isolated spindle cells with eosinophilic cytoplasm (Figure 2, $A$ ); immunohistochemical staining was positive for desmin (Figure 2, $B$ ). The final pathologic diagnosis was calcified fetal rhabdomyoma of the tricuspid valve and recurrent pulmonary embolism, with no evidence of tuberous sclerosis.

\section{DISCUSSION}

The clinical and pathologic features of the 2 sibling patients herein are very uncommon. Considering the frequency of rhabdomyoma as cause of fetal and neonatal primary cardiac tumor, ${ }^{1}$ findings of large, hyperechogenic intracardiac masses suggested rhabdomyoma as presumptive diagnosis in both patients. On the contrary, tricuspid valve location, absence of tuberous sclerosis, ${ }^{1,2}$ no tendency toward regression, severe intracardiac obstruction with repeated pulmonary embolization, and, most unusually, familial recurrence brought the latter diagnosis into question. Although 

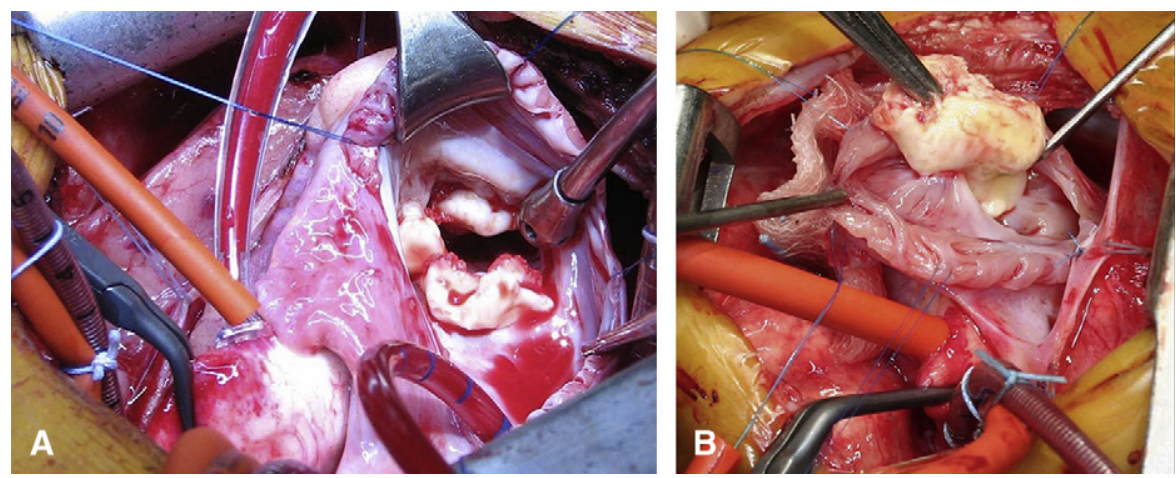

FIGURE 1. A, Operative view of the 3 tricuspid valve masses via right atriotomy in patient 1 . All 3 valve leaflets were encased in firm, amorphous sclerocalcified masses. B, Operative view of the tricuspid valve masses via right atriotomy in patient 2 . A single, large, and friable sclerocalcified mass, adherent to the right atrial free wall and obstructing almost completely the tricuspid valve orifice, was present. Residual tricuspid valve tissue was barely visible.

association with tuberous sclerosis approaches $100 \%$ of multiple rhabdomyoma cases, the syndrome may become apparent later in life. ${ }^{1,2}$ In addition, regression of the lesions is not invariable. ${ }^{2}$ Finally, rare cases of severe intracardiac obstruction requiring rescue excision have been documented. ${ }^{3}$ Diagnosis, however, became dubious only when histology of the three tricuspid valve masses in the older brother failed to identify any tumor cells, leaving only causes of neonatal dystrophic calcifications (amorphous cardiac tumor, endocarditis $)^{4, \mathrm{E} 1, \mathrm{E} 2}$ as likely etiologies. Consistent with this hypothesis, the family was not discouraged to face another pregnancy. The fetal ultrasound evidence of a nearly identical lesion in the younger brother, however, imposed reconsideration. Histologic diagnosis of fetal-type rhabdomyoma was eventually reached only in the latter sibling.

Fetal-type rhabdomyoma is a benign tumor described thus far only in extracardiac (skeletal muscle) locations. ${ }^{5}$ Contrary to the typical "spider" cell of cardiac rhabdomyoma, the histologic marker is the fetal myoblast ("spindle cell"'), presenting various degrees of differentiation. Pathophysiology of this tumor, when localized in the heart, is unknown. One can assume that given the clinical and pathologic similarities (including absent/poor cellularity, wealth of stromal content), the masses in the older brother were identical in nature, yet isolated at a more advanced stage of involution. The present experience with 2 preterm siblings suggests that the tumor underwent interruption of growth process shortly after birth, displaying tendency toward cellular necrosis and myxoid degeneration with dystrophic calcifications. Explanations for this behavior may include mummification-like regression due to unique susceptibility of tumor cells to hypoxia-ischemia, as postulated for cardiac rhabdomyoma by Wu et al. ${ }^{2}$ Accordingly, both neonates suffered from prolonged and profound hypoxia due to severe right-heart obstruction and shed pulmonary embolism, which were in turn caused by the tumor. Therefore, although fetal rhabdomyoma exhibited a self-limiting growth pattern, this very behavior resulted in a clinically malignant course during early infancy as characterized by lifethreatening hypoxic and pulmonary hypertensive crises. Whether frequency of fetal rhabdomyoma of the heart may be higher due to tendency toward dystrophic calcification, thereby explaining some cases of unexplained intrauterine demise, is at present entirely speculative. Interestingly, the
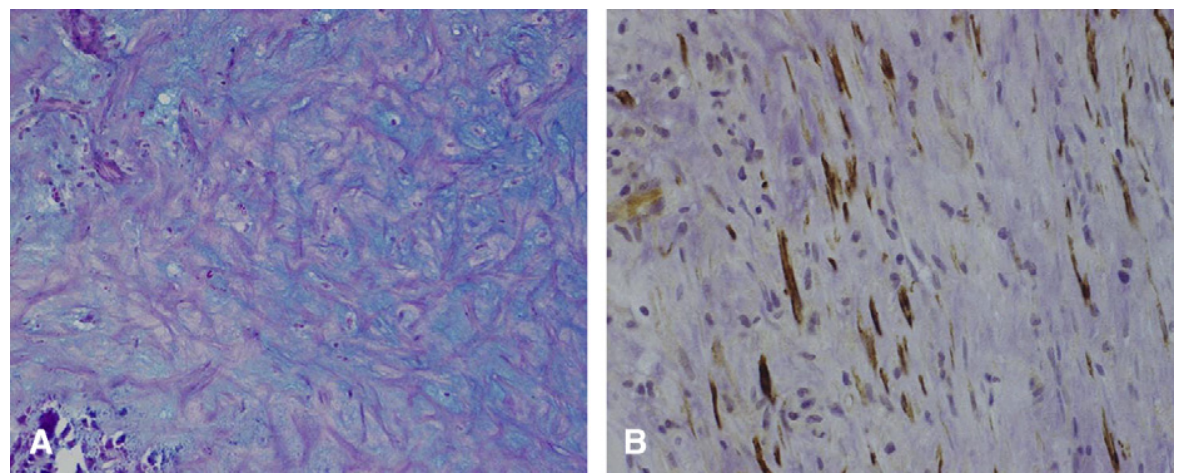

FIGURE 2. A, Alcian blue staining of the tumor of patient 2 showing small cellular areas consisting of a fibromyxoid stroma with isolated, spindlelike cells with eosinophilic cytoplasm compatible with fetal myoblasts. There was no evidence of "spider cells" typical of cardiac rhabdomyoma. B, Immunohistochemical staining of the tumor of patient 2 showed spindlelike cells positive for desmin. 
mother of the 2 siblings presented a history of multiple spontaneous abortions.

The management strategy of the patients herein was flexible due to unpredictable pathologic and clinical evolution of the tumor. At birth, PGE1 therapy and adequate atrial-level shunting, requiring atrial septostomy in 1 patient, were necessary due to univentricular physiology with ductal-dependent pulmonary blood flow. Unexpectedly, both neonates presented severe pulmonary hypertension, only partially responsive to intravenous and inhaled pulmonary vasodilators. Prematurity and persistent fetal circulation were thought to be responsible; only after postmortem diagnosis of patient 2 did we identify recurrent pulmonary embolism due to tumor calcification and fragmentation. Retrospectively, therefore, the rationale for delaying surgical excision (low body weight, prematurity, expectation of spontaneous regression) appears unjustified. On the contrary, intracardiac masses with clinicopathologic features inconsistent with cardiac rhabdomyoma must be referred for early surgical excision to allow for diagnosis and prevention of life-threatening embolism. Furthermore, when fetal-type rhabdomyoma is identified in a child, genetic counseling must consider the possibility of occurrence of the tumor in future pregnancies and should advise in utero echocardiographic screening. In the case of diagnosis, however, any decision to interrupt pregnancy should take into consideration the fact that radical resection in infancy is feasible and no association with tuberous sclerosis has been reported.

\section{References}

1. Isaacs H. Fetal and neonatal cardiac tumors. Pediatr Cardiol. 2004;25:252-73.

2. Wu SS, Collins MH, de Chadarévian JP. Study of the regression process in cardiac rhabdomyomas. Pediatr Dev Pathol. 2002;5:29-36.

3. Schreiber C, Vogt M, Kostolny M, Gunther T, Lange R. Surgical removal of a rhabdomyoma in a neonate as rescue therapy. Pediatr Cardiol. 2006;27: $140-1$.

4. Reynolds C, Tazelaar HD, Edwards WD. Calcified amorphous tumor of the heart (cardiac CAT). Hum Pathol. 1997;28:601-6.

5. Hansen T, Katenkamp D. Rhabdomyoma of the head and neck: morphology and differential diagnosis. Virchows Arch. 2005;447:845-54.

\title{
Diagnosis and management of tritruncal heart in an infant
}

\author{
Glenn J. Pelletier, MD, ${ }^{\mathrm{a}, \mathrm{b}}$ Mary Sokoloski, MD, ${ }^{\mathrm{c}}$ and Richard Kardon, DO, ${ }^{\mathrm{c}}$ Philadelphia, Pa, and Miami, Fla
}

Ventriculoarterial connections are typically arranged with the aorta and main pulmonary artery (MPA) joined to the left ventricle (LV) and right ventricle (RV), respectively. Until 1982, when Díaz-Góngora and colleagues ${ }^{1}$ reported an autopsy case in which 3 separate arteries arose from the base of the ventricles, tritruncal heart was an unknown defect. The third artery was an intermediate trunk that arose from the LV and continued on as the right pulmonary artery (RPA). We report the diagnosis and management of the first known living child to undergo successful repair of a tritruncal heart.

\footnotetext{
From the Department of Surgery, Drexel University College of Medicine, ${ }^{\mathrm{a}}$ and the Section of Cardiothoracic Surgery, St Christopher's Hospital for Children, ${ }^{\mathrm{b}}$ Philadelphia, Pa, and the Department of Pediatrics, Division of Pediatric Cardiology,

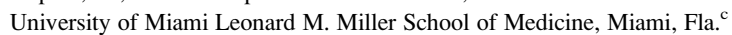
Received for publication Dec 4, 2007; accepted for publication March 13, 2008.

Address for reprints: Glenn J. Pelletier, MD, Section of Cardiothoracic Surgery, Saint Christopher's Hospital for Children, Erie Avenue at Front St, Philadelphia, PA 19134-1095 (E-mail: gpelleti@drexelmed.edu).

J Thorac Cardiovasc Surg 2009;137:753-5

0022-5223/\$36.00

Copyright (c) 2009 by The American Association for Thoracic Surgery

doi:10.1016/j.jtcvs.2008.03.057
}

\section{CLINICAL SUMMARY}

The patient was a male twin born at term. At 10 days after his birth, a heart murmur was detected. At presentation, the patient appeared well; however, the oxygen saturations recorded by noninvasive measurement varied from $83 \%$ to $97 \%$ when the patient was breathing ambient air. An echocardiogram demonstrated 3 arterial trunks originating from the base of the heart. The MPA $(11 \mathrm{~mm})$ arose from the $\mathrm{RV}$ and continued distally exclusively as the left pulmonary artery (LPA, $5 \mathrm{~mm})$. The aorta $(8 \mathrm{~mm})$ arose from the $\mathrm{LV}$ in the usual fashion. The third artery, the IT $(3 \mathrm{~mm})$, originated from the LV and continued as the RPA. At the base of the IT, a semilunar valve was seen through which antegrade flow into the RPA and regurgitant flow into the LV occurred (Figure 1,A). The RV pressure was estimated to be suprasystemic. There was predominantly right-to-left shunting through a patent foramen ovale. The LV was normal in size and function.

Cardiac catheterization confirmed the origin of the IT from the LV and its continuation as the RPA. Several aortopulmonary collaterals arose from the descending aorta to supply numerous segments of the right lung (Figure 1, $B$ ). Pressure measurements of the LV and RV were equivalent. 


\section{PATIENT 1}

The first patient was a $2.050-\mathrm{kg}$ male neonate, delivered preterm ( 36 weeks) via caesarean section for intrauterine growth restriction. The mother had a history of recurrent spontaneous abortion. Because of evidence of profound cyanosis the day after birth, the neonate was intubated and was started on PGE1 to ensure ductal patency and on inotropes to support the circulation. Chest radiograph showed decreased pulmonary vascular markings. Echocardiographic examination showed 3 large $(3 \times 5 \mathrm{~mm})$, hyperechogenic masses adherent to 3 leaflets of the tricuspid valve, causing severe valve stenosis. Blood cultures were negative. A presumptive diagnosis of cardiac rhabdomyomatosis was established. Due to restrictive shunting across the foramen ovale, balloon atrial septostomy was performed. In addition, evidence of rightto-left shunting at the level of ductus suggested persistent pulmonary hypertension. Therefore, inhaled NO therapy was started at $20 \mathrm{ppm}$ and subsequently combined with epoprostenol infusion and high-frequency ventilation. Weaning from inhaled $\mathrm{NO}$ and epoprostenol was achieved on days 13 and 20, respectively, by replacing them with bosentan and sildenafil. The infant was kept for 62 days in the intensive care unit and for 15 days in the ward. At the time of discharge, he was in satisfactory clinical condition, under sildenafil, bosentan, and diuretic medication. Predischarge echocardiogram showed growth of tricuspid valve masses, with moderate stenosis and mild insufficiency, bidirectional shunting at the atrial level, and residual mild pulmonary hypertension (peak $35 \mathrm{~mm} \mathrm{Hg}$ ). Neurologic assessment, electroencephalogram, magnetic resonance imaging, and abdominal ultrasound were unremarkable, making diagnosis of tuberous sclerosis unlikely. Due to echocadiographic evidence of no regression of tricuspid valve masses, at 11 months of age, the child was scheduled for surgical excision and closure of the atrial septal defect, to prevent particulate embolization and to obtain histologic diagnosis. The intraoperative finding was of $3 \mathrm{firm}$, large $(6 \times 7,4 \times 5$, and $6 \times$ $10 \mathrm{~mm}$ ) masses on the atrial surface of all 3 leaflets of the tricuspid valve (Figure 1, A), with dystrophic calcification and ulceration. The chordae tendineae were free, but the mass on the septal leaflet infiltrated the tricuspid valve annulus (Figures 1E, 2E). Radical excision was accomplished by shaving the masses off the anterior and posterior leaflets and removing the latter mass with part of the septal leaflet (Figure 2E), replacing it with a patch of autologous pericardium. The atrial septal defect was also patched with pericardium. Transesophageal echocardiogram showed successful repair with only mild valvular insufficiency. The infant was discharged after 7 days in excellent clinical condition, under no medications. Histologic examination of the excised masses showed connective-sclerotic nodules with nuclei of calcification- ossification and absence of tumor cells. A presumptive diagnosis of dystrophic calcification due to inflammatory disease (fetal endocarditis) or amorphous cardiac tumor was made. The patient is now a thriving 21-month-old with echocardiographic findings compatible with no mass recurrence, mild tricuspid insufficiency, and normal pulmonary artery pressure. Considering the presumptive diagnosis, genetic counseling did not discourage future pregnancies.

\section{PATIENT 2}

The younger brother was a 1.8-kg child also delivered preterm (33 weeks) via caesarean section for fetal bradycardia after antenatal ultrasound diagnosis of tricuspid valve masses during second trimester of gestation. Upon birth, the patient required ventilation for cyanosis and PGE1 infusion. Echocardiographic findings confirmed a large tricuspid mass $(16 \times 18 \mathrm{~mm})$ causing severe stenosis, left-to-right shunting at ductal level, and right-to-left shunting at atrial level through a nonrestrictive atrial septal defect. On day 13 , due to recurrent hypoxic spells and evidence of pulmonary hypertension, intravenous epoprostenol and oral sildenafil plus bosentan were started to improve oxygen saturation. Due to failure to respond, inhaled NO was also added. On day 21, the patient had a new hypoxic spell; based on evidence of restrictive left-to-right shunting across the ductus, the $2.0-\mathrm{kg}$ infant was referred for urgent operation. Intraoperative finding was a huge $(1.5 \times 2 \mathrm{~cm})$, crumbly, sclerocalcified mass, replacing the whole posterior and one third of anterior leaflet of the tricuspid valve, infiltrating annulus, papillary muscles, and trabeculae of the right ventricle (Figures 1, B, 3E, 4E). Radical excision of the mass was performed, and a large "sail-like" pericardial patch was used to replace the anterior and posterior leaflets (Figure 5E). The atrial septal defect was left. Weaning from extracorporeal circulation was impossible due to profound hypoxia, despite addition of a central aortopulmonary shunt (3-mm polytetrafluoroethylene graft), maximal ventilatory support, and inhaled NO $20 \mathrm{ppm}$, which also proved insufficient. The infant was hence placed on venoarterial ECMO and weaned after 3 days, using high $\mathrm{FIO}_{2}$ and inhaled NO therapy. Due to intercurrent infection and renal failure, inotropic support and peritoneal dialysis were started. On day 9, the patient died of irreversible hypoxic-hypercapnic respiratory failure. Necropsy showed multiple hemorrhagic infarctions of both lungs due to thromboembolic occlusions of distal pulmonary arterial branches. Lesions were both recent and old, some with partial thrombi recanalization. No evidence of tuberi was found. Histologic examination of the tumor showed an amorphous, calcified, slightly basophilic mass, with areas of mineralization and nuclei of calcification. Small cellular areas consisted of a fibromyxoid stroma and isolated, 
spindlelike cells with eosinophilic cytoplasm with areas of thickening (Figure 2, A); immunohistochemical staining was positive for desmin (Figure 2, B) and smooth-muscle actin. The pathologic diagnosis was consistent with calcified fetal rhabdomyoma of the tricuspid valve and recurrent pulmonary thromboembolism.

\section{References}

E1. Menahem S, Robbie MJ, Rajadurai VS. Valvar vegetations in the neonate due to fetal endocarditis. Int J Cardiol. 1991;32:103-5.

E2. Haug K, Kohlschmidt N, Dereser-Dennl M, Zielinski J, Merz E, Schafer D Familial fatal fetal cardiomyopathy with isolated myocardial calcifications: a new syndrome? Am J Med Genet. 2001;101:255-8. 


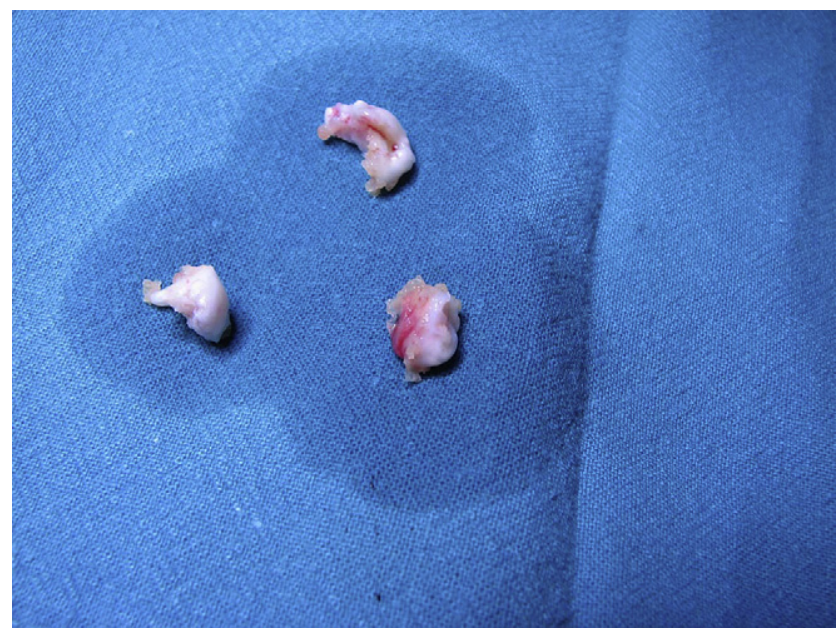

FIGURE E1. Gross anatomic aspect of the 3 masses in patient 1 . The lesions were large $(6 \times 7,4 \times 5$, and $6 \times 10 \mathrm{~mm})$ and sclerotic with visible areas of calcification, ulceration, and hemorrhage. 


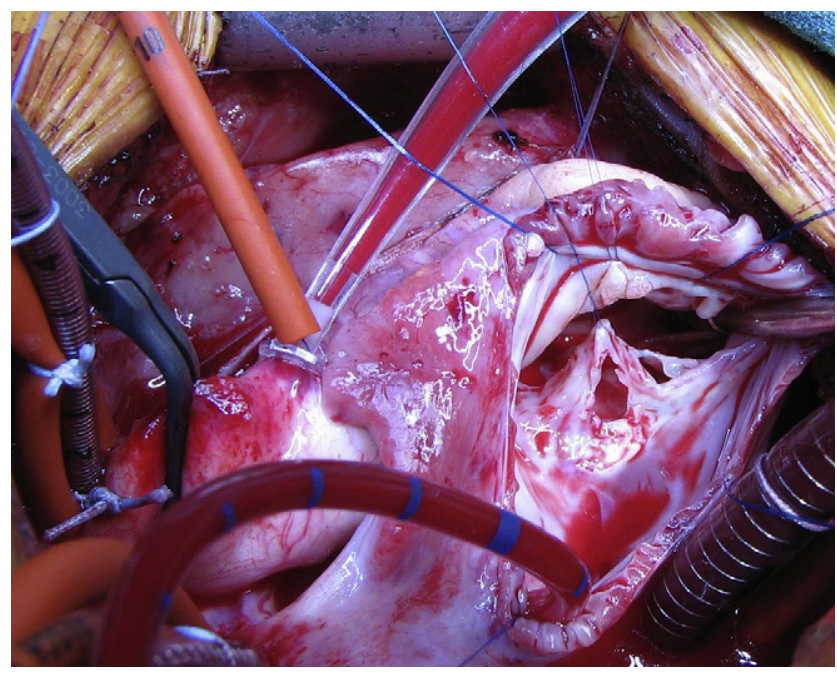

FIGURE E2. Operative view after complete excision of the 3 masses in patient 1. A large tissue defect in the septal leaflet and annulus of the tricuspid valve, where the septal mass was anchored, was evident. Due to infiltration of muscle and valve tissue, the latter mass required excision with its broad base of implant. 


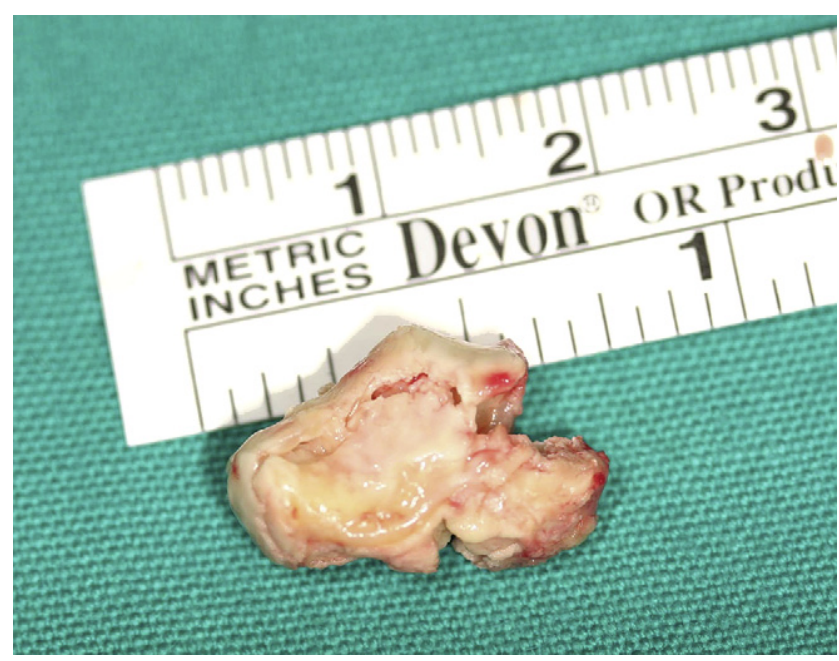

FIGURE E3. Gross anatomic aspect of the mass excised in patient 2 on a caliper to detail dimensions. Similar to the sibling patient, areas of calcification, ulceration, and hemorrhage were visible. 


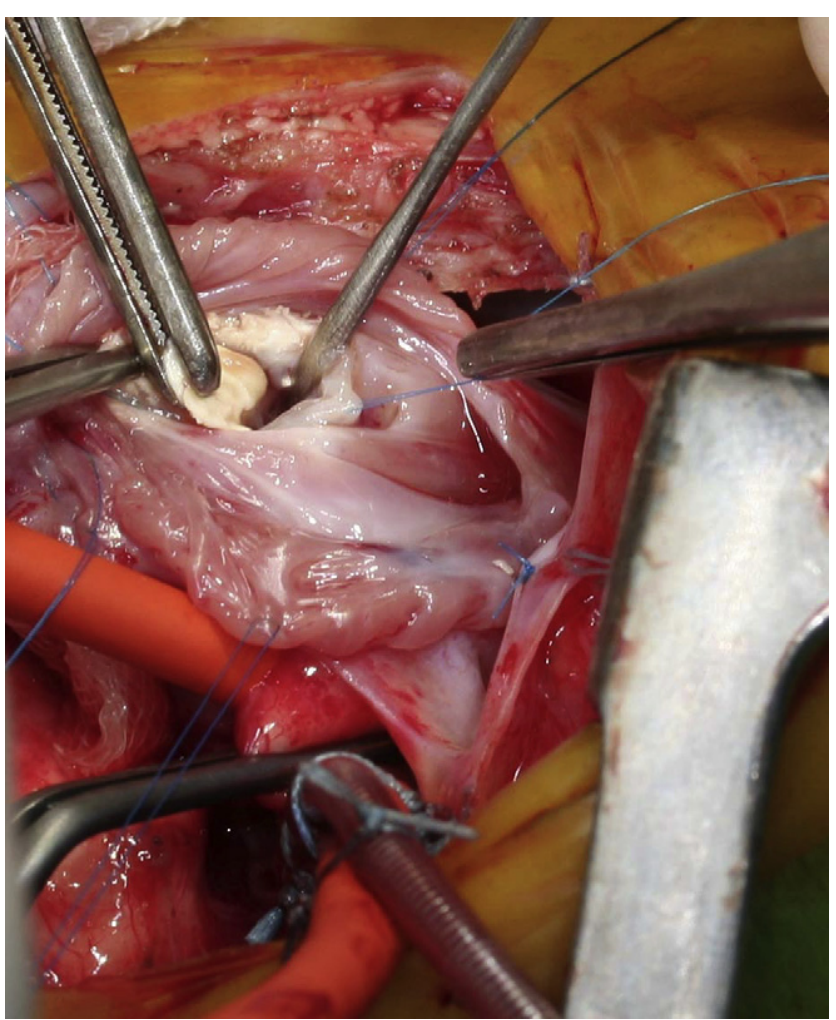

FIGURE E4. Operative view of patient 2 after excision of the mass with the whole posterior and one third of anterior leaflet of the tricuspid valve. The septal leaflet was intact and retracted to expose the infiltration of annulus, papillary muscles, and trabeculae of the right ventricle. 


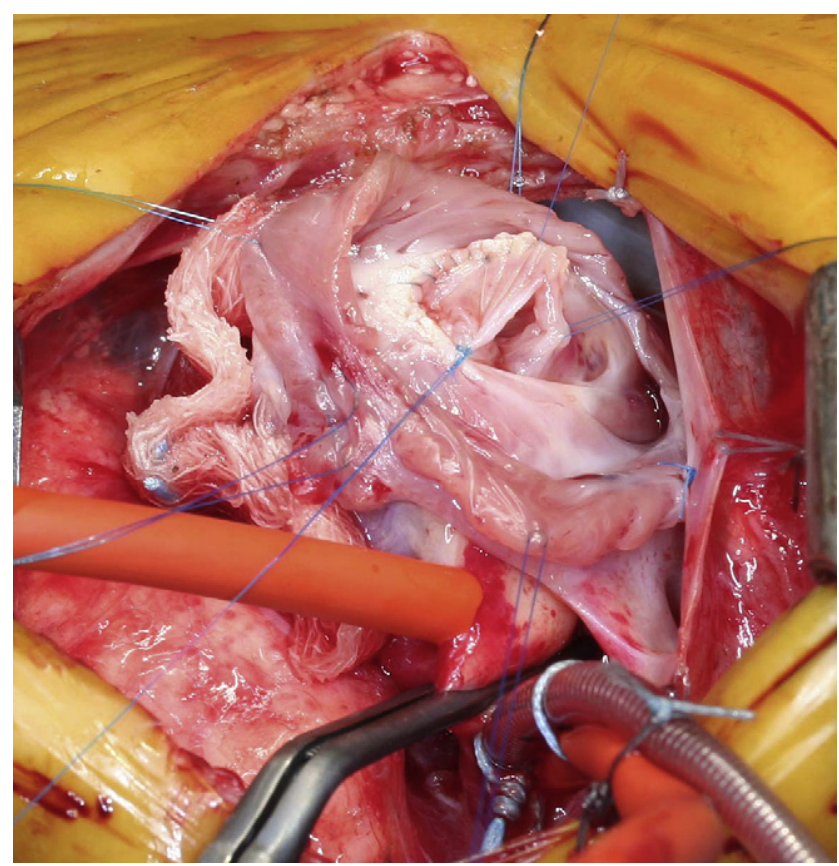

FIGURE E5. Operative view of the completed tricuspid valve repair in patient 2 . The anterior and posterior tricuspid leaflets were reconstructed using a large "sail-like" patch of tanned autologous pericardium. 